\title{
Thrombosed vascular incarcerated umbilical hernia- an unusual complication of chronic liver diseases
}

\begin{abstract}
Umbilical hernias are common in patients with chronic liver disease, caused by increased intra-abdominal pressure. In some patients the hernia contents can be the dilated paraumbilical vein. Complications are ulceration of the skin and vascular rupture, with high mortality. It can also occur thrombosis of paraumbilical herniated vein. Imaging tests are essential for the diagnosis of the contents of the sac in order to assist the treatment. Hepatocellular carcinoma is the most common primary tumor of the liver. Affects three times more men than women. The main risk factor is chronic liver disease. The imaging methods are essential for early diagnosis and to decrease morbidity and mortality. The authors report a case of a Thrombosed vascular incarcerated umbilical hernia with an MRI and ultrasound imaging tests diagnosis.
\end{abstract}

Keywords: liver diseases, carcinoma, umbilical, ultrasonography, magnetic resonance imaging
Volume 3 Issue 5 - 2017

\begin{abstract}
Márcio Luís Duarte,' Fabricius André Lyrio Traple, ${ }^{2}$ Thaís Nogueira Dantas, ${ }^{2}$ Egídio Cuzziol, ${ }^{2}$ Luiz Carlos Donoso Scoppetta ${ }^{2}$

'Department of Radiology,Webimagem, Brazil

${ }^{2}$ Department of Radiology, Hospital São Camilo, Brazil
\end{abstract}

Correspondence: Márcio Luís Duarte,Webimagem, Avenida Marquês de São Vicente 446, São Paulo, São Paulo, Brazil, Tel 55xx | 398 | | | 2799, Email marcioluisduarte@gmail.com

Received: October 24, 2017 | Published: November 15, 2017
Abbreviations: $\mathrm{CT}$, computed tomography; MRI, magnetic resonance imaging; ICU, intensive care unit

\section{Introduction}

Hepatocellular carcinoma is the most common primary tumor of the liver. Affects three times more men than women. ${ }^{1,2}$ The main risk factor is chronic liver disease. The imaging methods are essential for early diagnosis and to decrease morbidity and mortality.

The umbilical vein becomes the teres ligament after birth. In portal hypertension, collateral veins can rechannel the umbilical vein, forming a portosystemic shunt, called paraumbilical vein. The collateralization of the portal system with paraumbilical vein, periumbilical veins of the anterior abdominal wall and the superficial and deep epigastric veins, forming a network of dilated veins in the abdominal wall (aspect called "caput medusa") in patients with chronic liver disease and portal hypertension is known as CruveilhierBaumgarten syndrome.,

\section{Case presentation}

59 years old man with confusion, dyspnea and abdominal pain for 2days. History of hepatocellular carcinoma awaiting liver transplantation. CT scan performed 7 months earlier shows chronic liver disease with hepatic nodules, splenomegaly, dilatation of portal, splenic and superior mesenteric veins, and collateral circulation in the topography of gastric, splenic and perisplenic veins. There is recanalization of the paraumbilical vein that insinuates through the umbilical hernia with fat content.

MRI performed 4months after the CT scan shows signs of chronic liver disease with juxtaposing hepatic nodules with peripheral enhancement, associated to satellite nodules and splenomegaly; increased caliber of portal, splenic and superior mesenteric veins, with recanalization of paraumbilical vein, inferring portal hypertension (Figure 1)

Ultrasound performed in attendance reveals ascites, signs of chronic liver disease and portal hypertension, such as splenomegaly, dilated splenic and portal veins, and recanalization paraumbilical vein that presents with herniated fat content. This vascular herniated segment presents thrombosed (Figure 2). After the ultrasound, the umbilical hernia was reduced manually, and patient was removed to the ICU.
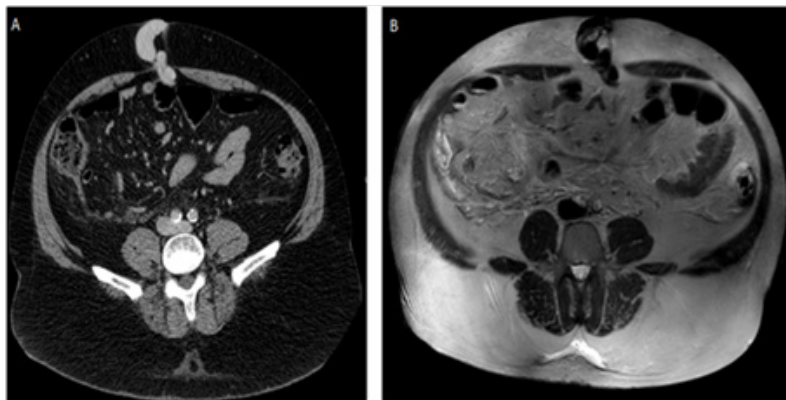

Figure I In A, Axial CT scans with contrast and in B, MRI without contrast in the axial T2 plane shows recanalization of paraumbilical vein insinuating through the umbilical hernia.
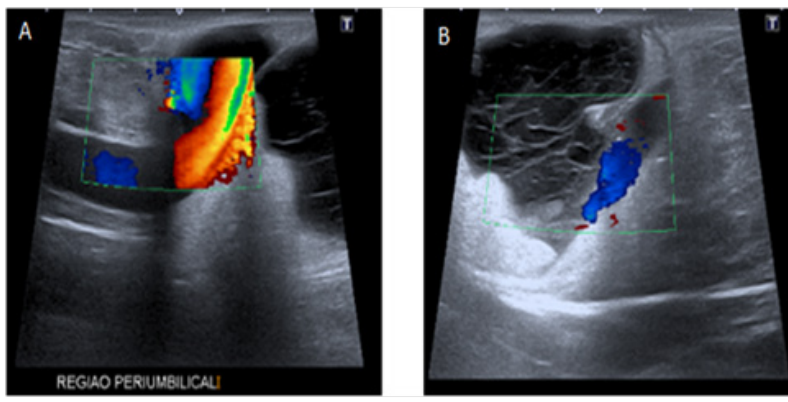

Figure 2 In $A$, ultrasound demonstrating recanalization of the herniated paraumbilical vein. In B, ultrasound demonstrating herniated paraumbilical vein segment without vascularization to Doppler - thrombosis.

\section{Discussion}

The umbilical vein is echogenic and becomes anechoic after recanalization, producing the appearance of "bull's eye" in the 
transverse plane of ultrasound and with a vascular channel larger than three millimeters in diameter is a specific sign of portal hypertension. ${ }^{4}$ If there is herniation of paraumbilical vein, Doppler ultrasound shows the paraumbilical vein protruding through the umbilical channel during the Valsalva maneuver. ${ }^{5}$

CT scan demonstrates dilated and tortuous paraumbilical vein, coming from the left branch of the portal vein, which runs through the falciform ligament toward the belly button, forming a network of periumbilical dilated veins-the blood possibly drains into the systemic circulation through the superficial and deep epigastric veins, reaching the external iliac vein. ${ }^{5}$

Umbilical hernias are common in patients with chronic liver disease, caused by increased intra-abdominal pressure. In some patients the hernia contents can be the dilated paraumbilical vein. ${ }^{6}$ Complications are ulceration of the skin and vascular rupture, with high mortality. It can also occur thrombosis of paraumbilical herniated vein. Imaging tests are essential for the diagnosis of the contents of the sac in order to assist the treatment. ${ }^{6}$

\section{Acknowledgements}

None.

\section{Conflict of interest}

The authors declare that there is no conflict of interests regarding the publication of this paper.

\section{Patient consent}

The written informed consent of the patient was obtained, for the publication of her case.

\section{References}

1. Schafer DF, Sorrell MF. Hepatocellular Carcinoma. Lancet. 1999;353(9160):1253-1257.

2. Kanematsu T, Sonoda T, Takenaka K, et al. The value of ultrasound in the diagnosis and treatment of small hepatocellular carcinoma. Br J Surg. 1985;72(1):23-25.

3. Vilgrain V, Lagadec M, Ronot M. Pitfalls in liver imaging. Radiology. 2016;278(1):34-51.

4. Matushita JPK, Laurentys-Medeiros J, Matushita JS, et al. Síndrome de Cruveilhier-Baumgarten. Radiol Bras. 2007;40:56-57.

5. Arora A, Mukund A, Dev A, et al. I can't change the direction of the wind, but I can adjust my sails to always reach my destination: CTportography depiction of different Hepatofugal Collaterals in Cirrhosis. ECR 2013. Poster No: C-1135. 2013.

6. McAlister V. Management of umbilical hernia in patients with advanced liver disease. Liver Transpl. 2003;9(6):623-625. 